\title{
Quantum Nucleation of Phase Slips in a 1d Model of a Superfluid
}

\author{
Jose A. Freire, Daniel P. Arovas and Herbert Levine \\ Physics Department, University of California, San Diego \\ La Jolla, CA 92093
}

(August 14, 2018)

\begin{abstract}
We use a $1 d$ model of a superfluid based on the Gross-Pitaevskii Lagrangian to illustrate a general numerical method designed to find quantum tunneling rates in extended bosonic systems. Specifically, we study flow past an obstacle and directly solve the imaginary time dynamics to find the "bounce" solution connected with the decay of the metastable laminar state via phase slip nucleation. The action for the tunneling configuration goes to zero at the threshold (in superfluid velocity) for classical production of these slips. Applications to other processes are briefly discussed.

PACS numbers: 64.60.Qb, 67.40.-w, 67.40.Vs
\end{abstract}

The subject of quantum tunneling in extended systems continues to be of great interest in a variety of condensed matter applications. Examples include superflows (either superfluids or superconductors) in which dissipation can be induced by the creation of phase slips via tunneling. Often, the rates of these processes are calculated by focusing on one relevant degree of freedom (perhaps the center-point of a specific ansatz for the quantum field) and approximately integrating out the remainder of the degrees of freedom; this approach thereby reduces the problem to quantum mechanics of a single particle. This reduction precludes one from understanding the extent to which re-arrangement of the (usually nonlinear) field degrees of freedom can lead to more advantageous tunneling paths.

In this work, we offer a more direct approach to the evaluation of quantum tunneling rates in extended bosonic systems. Specifically, we use the idea popularized by Coleman and Callan that quantum tunneling can be studied by finding a specific solution to the classical equations of motion continued to imaginary time, the socalled "bounce". To illustrate our method, we explicitly study the nucleation of phase slips in a $1 \mathrm{~d}$ model of a superfluid flowing past an obstacle. We show how the bounce solution can be constructed, how it determines the nucleated state of the entire field and how one can compute the action for this solution, thereby finding the semiclassical rate. At the end, we discuss extensions to other bosonic systems as well as comment on the modifications that will be required to study fermions.

To model a superflow with velocity $v$ past a $1 d$ obstacle we use the Gross-Pitaevskii Lagrangian density [1]

$\mathcal{L}=i \hbar \bar{\psi} \partial_{t} \psi-\frac{\hbar^{2}}{2 m}\left|\partial_{x} \psi\right|^{2}-\frac{1}{2} g\left(|\psi|^{2}-\mu(x) / g\right)^{2}-i \hbar v \bar{\psi} \partial_{x} \psi$

The first three terms arise directly from considering bosons interacting with each other via $\delta$ function potentials of strength $g$, in the presence of an obstacle treated as a position-dependent chemical potential $\mu(x)$. The last piece comes from working in a frame in which the obstacle is at rest. We will explicitly choose $\mu(x)$ to have the form of a square well of width $\Delta$ and depth $\eta \mu_{0}(\eta<1), \mu_{0}$ being the uniform chemical potential outside; this will give rise to a depletion of the condensate density in the well. In this model, the natural unit of length is the coherence length $\xi=\hbar / \sqrt{m \mu_{0}}$, of velocity is the sound velocity, $c=\sqrt{\mu_{0} / m}$, and of condensate amplitude is $\sqrt{\rho_{0}} \equiv \sqrt{\mu_{0} / g}$. A dimensionless parameter, $\alpha \equiv \rho_{0} \xi^{d}(d=1$ here), analogous to $1 / \hbar$, then determines the importance of quantum fluctuations; we assume $\alpha \gg 1$. The classical dynamics derived from the above Lagrangian is the Galilean-transformed nonlinear Schrödinger equation (NLSE). In natural units, with $\left(\zeta(x)=\mu(x) / \mu_{0}\right)$,

$$
i \partial_{t} \psi=-\frac{1}{2} \partial_{x}^{2} \psi+\left(|\psi|^{2}-\zeta(x)\right) \psi-i v \partial_{x} \psi .
$$

In this equation, $\psi$ is often interpreted as representing "the wavefunction of the condensate". The spatial boundary condition is taken to be one where the $\psi$ tends to a unimodular constant far away from the obstacle.

In recent work by Frisch et al [2], the NLSE in two spatial dimensions was utilized to study the problem of $2 d$ superflow past a circular obstacle. They found that there exists a critical velocity such that for $v<v_{\mathrm{c}}$, one is able to find time-independent solutions corresponding to a laminar flow around the obstacle, whereas for $v>v_{\mathrm{c}}$ only time-dependent solutions exist. The latter solutions correspond to a superflow that periodically nucleates vortex-antivortex pairs in the proximity of the disk; these pairs are subsequently advected by the flow. Similar results were found in Ref. [3] for a superflow in a $2 d$ channel containing a constriction. The $1 d$ analog of these vortex-antivortex pairs are phase slips. These are events localized in both space and time where the amplitude of the condensate goes to zero and the phase slips by $2 \pi$. Accordingly, we investigated numerically the existence of time-independent solutions of this equation; this was done by writing the time-independent NLSE in 
a $1 d$ grid and using Newton's method complemented by Powell's hybrid method [4,5] to solve the resulting system of nonlinear algebraic equations. In direct analogy to the $2 \mathrm{~d}$ findings, we found that, associated with each well depth and width, there is a critical value of the superflow velocity, $v_{\mathrm{c}}$, above which such solutions do not exist.

The above result implies that the solution of the NLSE above $v_{\mathrm{c}}$ is time-dependent. To find the nature of this solution, the time-dependent NLSE was integrated using an operator splitting method [6] accurate to $\mathcal{O}(d t)$ and unconditionally stable. An example of what we found is shown in Fig. 1. We observed the nucleation of the phase slip, typically at the edge of the well where $\left|\partial_{x} \zeta\right|$ is greatest, and the subsequent emission of a soliton. This soliton, which propagates outside the well, is a member of a family of solutions of the NLSE with $\zeta=1$ [7]. Up to an overall phase,

$$
\psi_{\mathrm{u}}(x, t)=\sqrt{1-u^{2}} \tanh \left[\sqrt{1-u^{2}}[x-(u+v) t]\right]+i u .
$$

This is a "dark" soliton: the amplitude dips to $|u|$ at its center. It carries a momentum $P=-2 u \sqrt{1-u^{2}}$ and accounts for a phase slip of $\pi-2 \sin ^{-1} u$ (see Fig. 1).

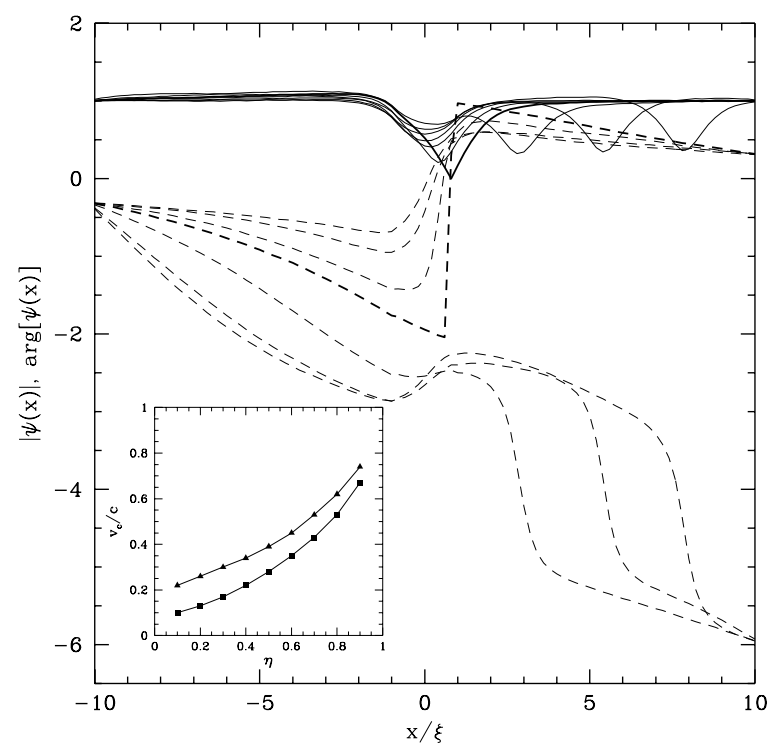

FIG. 1. The time evolution for $v>v_{\mathrm{c}}$, showing amplitude (solid line) and phase (dashed line) of $\psi$ at 7 times between $2 \xi / c$ and $24 \xi / c$. The phase slip occurs at $t \approx 12 \xi / c$, precisely at the right edge of the square well. Afterwards, a soliton is propagates to the right. The well width was $1.6 \xi$ and the chemical potential inside was $0.3 \mu_{0}$. The velocity was $v=0.4 c>v_{\mathrm{c}}=0.3 c . \psi$ was fixed at the ends. Inset: critical velocity versus well depth for wells of width $3.2 \xi$ (squares) and $1.6 \xi$ (triangles).

The existence of time-independent solutions of the NLSE only for $v<v_{\text {c }}$ suggests a picture where the parameter $v$ creates a barrier that prevents the classical decay of the metastable, time-independent state [8]. This barrier disappears when $v>v_{\mathrm{c}}$ and thus only timedependent solutions can be found. However, the steady flow state is only metastable and therefore can decay via quantum fluctuations. This is similar to the "fate of the false vacuum" problem studied by Coleman and Callan [9]. One can therefore use their formalism which relates the the tunneling rate (to leading order in a semiclassical expansion) to the classical action of the periodic "bounce solution", which proceeds in imaginary time from the metastable state to the nucleated field and back again. With the exception of one paper which studies nuclear fission 10], the possibility that one could directly solve for these bounce solutions does not seem to have been previously realized.

In detail, we consider a coherent state path integral representation of the matrix element of the evolution operator for imaginary time,

$$
\left\langle\psi_{0}\left|e^{-\mathcal{H} T / \hbar}\right| \psi_{0}\right\rangle=\int_{\substack{\psi(-T / 2)=\psi_{0} \\ \bar{\psi}(+T / 2)=\psi_{0}^{*}}} \mathcal{D}[\psi, \bar{\psi}] \exp \left[-S_{\mathrm{E}}[\psi, \bar{\psi}] / \hbar\right]
$$

Here, $\left|\psi_{0}\right\rangle$ is the metastable coherent state wavefunction. The Euclidean action is ( $L$ is the spatial size of the system)

$$
\begin{aligned}
& S_{\mathrm{E}}[\psi, \bar{\psi}]=\rho_{0} \xi \int_{-T / 2}^{T / 2} d \tau \int_{-L / 2}^{L / 2} d x\left[\bar{\psi} \partial_{\tau} \psi+E(\bar{\psi}, \psi)\right] \\
& E(\bar{\psi}, \psi)=\frac{1}{2}\left(\partial_{x} \bar{\psi}\right)\left(\partial_{x} \psi\right)+\frac{1}{2}[\bar{\psi} \psi-\zeta(x)]^{2}-i v \bar{\psi} \partial_{x} \psi
\end{aligned}
$$

The bounce solution is a classical configuration which extremizes this action; we easily obtain the bounce equations for the fields $\psi$ and $\bar{\psi}$ :

$$
\begin{aligned}
-\partial_{\tau} \psi & =-\frac{1}{2} \partial_{x}^{2} \psi+[\bar{\psi} \psi-\zeta(x)] \psi-i v \partial_{x} \psi \\
\partial_{\tau} \bar{\psi} & =-\frac{1}{2} \partial_{x}^{2} \bar{\psi}+[\bar{\psi} \psi-\zeta(x)] \bar{\psi}+i v \partial_{x} \bar{\psi}
\end{aligned}
$$

with to $\psi(-T / 2)=\psi_{0}$ and $\bar{\psi}(T / 2)=\psi_{0}^{*}$. The spatial boundary conditions are the same as before. These equations imply $\bar{\psi}(\tau)=\psi(-\tau)^{*}$, hence $\bar{\psi}(\tau)=\psi^{*}(\tau)-$ the density $\bar{\psi} \psi$ is real - only at three time slices: $\tau= \pm \frac{1}{2} T$, when $\psi=\psi_{0}$, and $\tau=0$, when the field emerges from the barrier and the phase slip has been nucleated 11. It is crucial to recognize that we do not need to make any assumption regarding the nature of the nucleated state, as the solutions of the equations will directly and uniquely determine this field configuration.

These equations can not be solved by integrating forward in time. This is because the $\bar{\psi}$ field has negative diffusivity and hence forward in time integration is illposed; alternatively, eliminating $\bar{\psi}$ using the above relation will give an explicitly non-local (in time) system. 


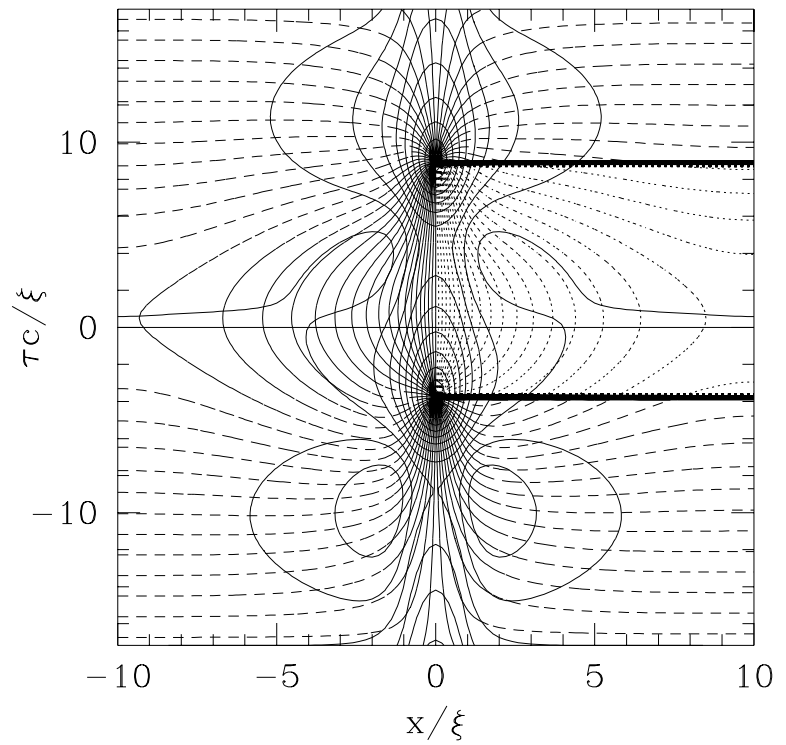

FIG. 2. Contour plot of the amplitude (solid) and phase (dashed) of $\psi(x, \tau)$ for the bounce solution. Here $v=0.1 c$ while $v_{\mathrm{c}}=0.3 \mathrm{c}$. Heavy lines are where the phase crosses $-2 \pi$ and do not correspond to any singularity. The well parameters are the same as in Fig. 1.

Even were this not to be the case, the fact that the bounce is a saddle-point as opposed to a minimum renders time-integration difficult. Instead, we solved those equations iteratively by discretizing them on a space-time lattice and using the Newton-Powell method mentioned above to find the zero of the resulting system of nonlinear algebraic equations. This iterative scheme does not care about time-locality, nor does it assume stability.

In solving the bounce equations on a space-time lattice one must choose a time interval $T$ long enough to include all the structure around $\tau=0$, as it is that part of the bounce that contributes the most to the action and that gives the nucleated state. We set up the equations in such a way that at $\tau=-T / 2$ we had the exact metastable state, while at once we also enforced the relation $\bar{\psi}(\tau)=\psi^{*}(-\tau)$. Because $T$ is finite, the solution obtained is not exactly periodic $-\psi$ at $\tau=T / 2$ is slightly different from $\psi$ at $\tau=-T / 2$ - but the difference can be made arbitrarily small by increasing $T$. The energy of the solution, however, was nearly constant and equal to the metastable state energy, as is the case for the true bounce solution. We also work with a finite spatial size. Whereas in the infinite system size limit we can have both $\psi \rightarrow e^{i \theta_{0}}$ and $\partial_{x} \psi=0$ as $x \rightarrow \pm \infty$, in our case we had to choose between these two options. We found that for smaller system sizes the gradient condition worked best. Typically we had $T \sim 34 \xi / c$ and $L \sim 20 \xi$ in a grid of 100 spatial points by 50 time points. This resulted in a system of 10,000 real nonlinear algebraic equations for the real and imaginary parts of $\psi$. The iterative scheme converges rapidly to a well-behaved solution when an ini-

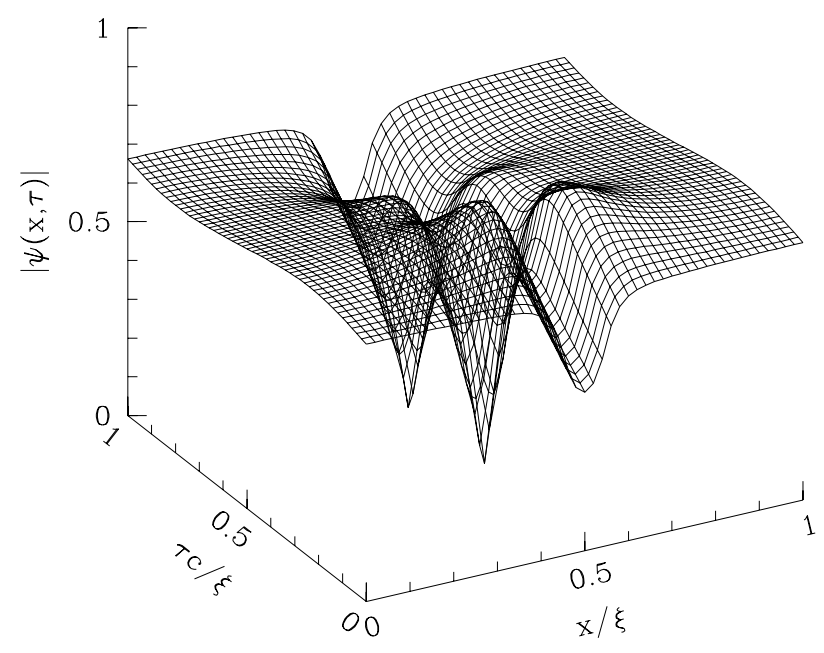

FIG. 3. The surface plot of the amplitude of the field $\psi$ of Fig. 2. The depletion of the condensate inside the well and in the vortex cores is apparent.

tial guess with the correct topology is used to start the process.

As expected, we found a solution that precisely corresponds to the quantum nucleation of a phase slip from the time-independent state of the given well at superflow velocity $\left(v<v_{\mathrm{c}}\right)$. The field $\psi(x, \tau)$ corresponding to a phase slipped state at $\tau=0$ has a singular point in negative time around which its phase winds counter-clockwise and another at positive time with clockwise phase winding. This vortex-antivortex pair in space-time results in a state at $\tau=0$ with a phase slip of $2 \pi$ with respect to the metastable state at $\tau= \pm T / 2$. The amplitude and phase of a typical solution $\psi$ are shown in Figs. 2 and 3. Note that the phase singularities are not symmetrically displaced about $\tau=0-\psi$ and $\bar{\psi}$ are only related by complex conjugation for $\tau=0, \pm T / 2$. Precisely at $\tau=0$ the configuration is interpretable as one for the ordinary (i.e. real-time) NLSE and hence can be treated as the initial condition for the subsequent real-time evolution of the nucleated phase slip. When this is done, the result is quite similar to what we have already seen occurs for the classically nucleated slip at $v>v_{\mathrm{c}}$; that is, a soliton is formed which propagates to the right, transferring energy from the obstacle region towards infinity.

The decay rate of the metastable state can be found using $\Gamma \propto \exp \left[-\Delta S_{\mathrm{E}} / \hbar\right]$, where $\Delta S_{\mathrm{E}}$ is the difference between the bounce action and the metastable state action. Since the dynamics in imaginary time conserves energy, we have 


$$
\Gamma \propto \exp \left(-\rho_{0} \xi \int d \tau \int d x \bar{\psi} \partial_{\tau} \psi\right)
$$

We solved the bounce equations for various superflow velocities with a given well and computed $\Gamma$ according to equation 5. The behavior is depicted in Fig. 4; note that the action approaches zero as we reach the classical threshold. As $v$ approaches $v_{\mathrm{c}}$ the vortex-antivortex pair separation decreases until eventually no time-dependent solution of equations 3 can be found for $v>v_{\mathrm{c}}$.

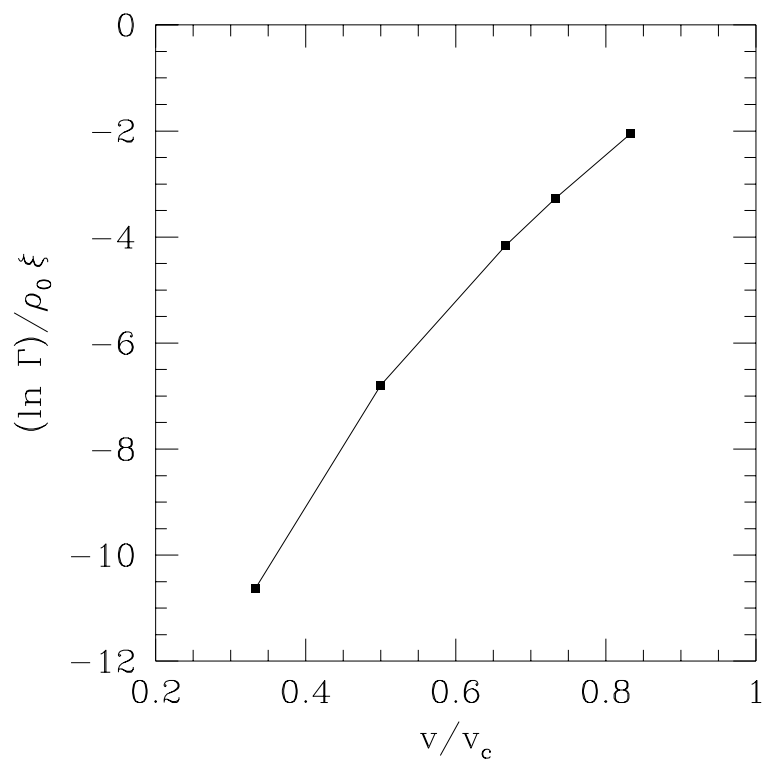

FIG. 4. An example of the dependence of the semiclassical decay rate on the superflow velocity. The well is the same as Figs. 2 and 3 . For $v>v_{\text {c }}$ no metastable state exists.

Although we have described our method for a specific example, it should be clear that the same approach can be used to compute tunneling rates and nucleated states for any bosonic system. Applications we have in mind include higher dimensional superfluid-obstacle problems (such as the quantum analog of the $2 \mathrm{~d}$ classical nucleation of vortex-antivortex pairs discussed above) and the decay of the metastable Bose-Einstein condensate state for trapped alkali atoms with attractive interactions [12]. One can also imagine straightforward extensions to the traditional field of macroscopic quantum tunneling where one could study the tunneling of, say, a two level system coupled to a nonlinear set of continuous degrees of freedom. Finally, extensions to fermionic systems (either charged or uncharged) are more challenging. Integrating out the fermions typically leaves one with a bosonic action in the form of some functional determinant and the resultant equation of motion involves successfully evaluating the inverse of a complicated operator. Whether it is feasible to directly find the bounce solution for this case (with no further approximations) is currently under investigation.

We wish to thank J. Koplik and S. Renn for valu- able discussions. HL is supported in part by NSF Grant DMR94-15460.

[1] E. P. Gross, J. Math. Phys. 4, 195 (1963); L. P. Pitaevskii, Sov. Phys. JETP 13, 451 (1961).

[2] T. Frisch, Y. Pomeau and S. Rica, Phys. Rev. Lett. 69, 1644 (1992).

[3] M. Stone and A. M. Srivastava, J. Low Temp. Phys. 102, 445 (1996).

[4] M. J. D. Powell, in Numerical Methods for Nonlinear Algebraic Equations, P. Rabinowitz ed. (Gordon and Breach, London, 1970).

[5] J. E. Dennis Jr. and R. B. Schnabel, Numerical Methods for Unconstrained Optimization and Nonlinear Equations, (Prentice Hall, New York, 1983).

[6] W. H. Press, S. A. Teukolsky, W. T. Vetterling and B. P. Flannery, Numerical Recipes (Cambridge University Press, Cambridge, 1986).

[7] V. E. Zakharov and A. B. Shabat, Sov. Phys. JETP 37, 823 (1973).

[8] The breaking of Galilean invariance, either by an impurity, or by the coupling to the electromagnetic field, is crucial. Both are relevant in superconductors - see e.g. J.M. Duan, Phys. Rev. Lett. 74, 5128 (1995); A. D. Zaikin, D. S. Golubev, A. van Otterlo, and G. T. Zimányi, Phys. Rev. Lett. 78, 1552 (1997); S. R. Renn and J.-M. Duan, Phys. Rev. Lett. 76, 3400 (1996).

[9] S. Coleman, Phys. Rev. D 15, 2929 (1977); C. G. Callan and S. Coleman, Phys. Rev. D 16, 1762 (1977).

[10] S. Levit, J. W. Negele and Z. Paltiel, Phys. Rev. C 22, 1979 (1980). This paper studies a nucleus consisting of a finite number of fermions and hence must deal with a very different form of the action than would occcur in any bosonic system. In addition, a much more involved iteration scheme was employed, as opposed to the direct Newton's iteration utilized in our work.

[11] If one treats the familiar problem of quantum tunneling potential $V(x)$ using coherent states, one obtains the equations in a $\partial_{\tau} z=-\partial_{\bar{z}} H, \partial_{\tau} \bar{z}=\partial_{z} H$ for $z=$ $(x+i p) / \sqrt{2}$ and $\bar{z}=(x-i p) / \sqrt{2}$. These dynamics conserve $H(z, \bar{z})$, so to keep $H$ constant, one must travel underneath the barrier, leading to imaginary $p$, i.e. $\bar{z} \neq z^{*}$. See, for example, J. K. Jain and S. Kivelson, Phys. Rev. B 37, 4111 (1988).

[12] P. A. Ruprecht, M. J. Holland, K. Burnett, and M. Edwards, Phys. Rev. A 51, 4704 (1995); H. T. C. Stoof, preprint cond-mat/9601150. 